\title{
Analysing the light curve and spectra of the first detected kilonova
}

\section{Anders Jerkstrand*t}

Max-Planck Institute for Astrophysics

E-mail: anders@mpa-garching.mpg.de

We report on the first clear detection of a kilonova; ejected radioactive debris from a neutron star merger. The transient AT2017gfo was detected 12h after GW170817 and associated short GRB170817A. It reached a peak UVOIR luminosity of $10^{42} \mathrm{erg} / \mathrm{s}$ on a time-scale of $\sim 1 \mathrm{~d}$. Modelling of the bolometric light curve suggests ejection of about $0.04 M_{\odot}$ of ejecta at $0.2 \mathrm{c}$. The ejecta has low opacity, indicating little lanthanide content. Spectroscopic modelling suggests identification of r-process elements Cs and Te. The combination of 2s delay between the GW and the GRB, a large ejecta mass, and low opacity all suggest dominance by a wind component and survival of a hypermassive neutron star for $t \sim 2 \mathrm{~s}$.

7th Fermi Symposium 2017

15-20 October 2017

Garmisch-Partenkirchen, Germany

* Speaker.

${ }^{\dagger}$ Based on a talk given for the whole team of Ref. 8 


\section{Introduction}

It has long been known that compact objects, neutron stars (NS) and black holes (BH), can merge by gravitational wave inspiralling. The result is a black hole, or possibly a very massive neutron star in some cases. Accretion processes in this event are leading candidates to produce short GRBs [1]. It has also long been thought that such mergers may eject a small amount of mass $[2,3]$. The physical conditions are such that this mass is neutron rich and mainly consists of r-process elements.

In the past 10-15 years research on this mass ejection process has accelerated as it has become increasingly possible to simulate the complex hydrodynamics and nucleosynthesis. Theoretical predictions have robustly converged upon ejection of $\sim 0.01 M_{\odot}$ of material at $\sim 0.1 c$, mainly consisting of r-process elements with electron fraction $Y_{e}=0.1-0.4$ (see e.g. [4] for a review). This has in turn led to an increased interest in whether this material may be detectable as some kind of supernova-like transient. The original speculation on this point was made by Li \& Paczynski 1998 ([5]) but not revived again until the work by Metzger et al. 2010 ([6]), carrying out the first detailed calculations. Metzger termed such a transient kilonova (KN).

The theoretical predictions have been building up to the possibility to detect $\mathrm{KNe}$ following mergers detected by LIGO and VIRGO. Following GW170817, the detection of two inspiralling neutron stars at a distance of only $40 \mathrm{Mpc}$ [7], several groups in the world pointed their telescope to the site and waited for what may come. And what came was AT2017gfo, now the topic of over a dozen papers in Science and Nature and countless in other journals. It has been estimated that $30 \%$ of the world-wide astronomical community was somehow involved in the study of this transient, and it is a landmark event in astronomy. Here I report on the optical/NIR study of AT2017gfo carried out by our team, with full publication of results in [8].

\section{The expectations: kilonova theory}

It was realized in the 1960s that even extremely powerful explosions of astrophysical objects may not be particularly bright in UVOIR radiation. If the object is compact, the photons cannot diffuse out and the nebula instead cools by adiabatic expansion. By the time the compactness is reduced so photons can escape, the internal energy has been degraded so much that not much energy is left to radiate [9]. For explosions of compact stars like white dwarfs or helium cores, virtually all the SN light is instead generated by continuous energy input by radioactive decay. For an explosion from a region the size of a neutron star, the adiabatic cooling is even more extreme and the transient would not be detectable unless extremely close to us.

Li \& Paczynski 1998 ([5]) were the first to point out that most elements created will be radioactive and thus provide an internal power source in analogy with Type I SNe $\left({ }^{56} \mathrm{Ni}\right)$. At that time the efficiencies of these radioactivities were not known, preventing a detailed prediction of the transient characteristics. Metzger et al. 2010 ([6]) provided the first such calculation, showing that the radioactivity robustly follows a $t^{-1.2}$ power law with a power level giving a transient at about $10^{42} \mathrm{erg} \mathrm{s}^{-1}$ at peak.

Much work since then has been focused on determining the opacity of the material, which has a strong effect on the appearance. Kasen et al. 2013 ([10]) made the important demonstration 
that lanthanides have much higher opacity than iron or lighter r-process elements. If such ejecta dominate, the peak will be reached at $\sim 10 \mathrm{~d}$ rather than $\sim 1 \mathrm{~d}$, and peak in the near infrared rather than in the UV/optical.

Linked to the opacity is a more detailed picture of the components of the ejected material. Two main components are delineated in current models; the dynamic and wind components. The dynamic component is directly ejected on a timescale of milliseconds and likely has a low $Y_{e}$. The wind arises from the accretion disk, is ejected on longer time-scales, and can have a higher $Y_{e}$. These components are predicted to have similar mass [11], and similar velocities, but uncertainties are significant.

\section{AT2017gfo}

Following the GW trigger of GW170817, our ePESSTO team launched a comprehensive monitoring programme involving 5 telescopes. The transient was imaged by four different telescopes; PS1, NTT (the ePESSTO telescope), the Boyden Telescope, and the GROND@2.2m telescope [12]. Full optical/NIR measurements were obtained at $+1.4 \mathrm{~d}$ by GROND and regularly after this epoch. The $0-1.4 \mathrm{~d}$ phase has more sparse coverage. PS1 gave $i z y$ magnitudes at $0.7 \mathrm{~d}$ and the Boyden telescope an r-band magnitude at $1.2 \mathrm{~d}$. All optical bands had faded below detectability by $7 \mathrm{~d}, J$ and $H$ had faded out at $11 \mathrm{~d}$, whereas $K_{s}$ detections were made up to $18 \mathrm{~d}$. A bolometric light curve was constructed by fitting a blackbody to the photometry and integrating between $1000 \AA-2.5 \mu \mathrm{m}$. At earliest phases, SWIFT data in $u v w 2, u v m 2, u v w 1$, and $U$ was also used to complement the NIR data (optical data is missing). A blackbody is a relatively good description of the photometry (see Fig 3. in [13]).

The bolometric light curve shows that the kilonova has peaked already at $t<0.6 \mathrm{~d}$, or is possibly peaking at $\sim 1 \mathrm{~d}$ (within uncertainties of the bolometric reconstruction). The peak luminosity is around $10^{42} \mathrm{erg} \mathrm{s}^{-1}$; as bright as a typical Type Ibc supernova. From this it was immediately clear that the transient had properties in rough agreement with predictions for $\mathrm{KNe}$ with relatively low opacity $[5,6]$.

\subsection{Light curve modelling}

In modelling transients the initial step is typically the bolometric light curve. Whereas the individual photometry depends on the detailed structure of the ejecta through the detailed location of the photosphere, the bolometric luminosity is governed by more simple principles. In the case of radioactivity-powered transients such as kilonovae this is diffusion.

A powerful semi-analytic method to model the bolometric light curve was developed by Arnett $1980,1982([14,15])$. In this framework the bolometric luminosity is determined by a time-integral over the power source function. While opacity has to be assumed constant in time and space for the case of radioactive energy input, the model does consider both diffusion and adiabatic cooling with a velocity-dependent temperature and density profile (homology assumed). There is no fundamental assumption about the nature of the power term in this formalism. In [16] and [17] this was exploited by applying the framework to other power sources than the classical ${ }^{56} \mathrm{Ni} /{ }^{56} \mathrm{Co}$ governing the vast majority of SNe. Here, we generalize the power term to a power law. 
Other semi-analytic formalisms exist. One is by Metzger 2017 ([18]). Here dynamic evolution is allowed, but the radiation transport is simplified with a decoupled sum of contributions by each shell. It is more flexible in that is allows opacity to vary by some prescribed manner; the method is in a sense more a simple numeric model than a semi-analytic one. In kilonovae dynamic evolution is negligible and one may argue that the Arnett method is preferable although this would need to be tested against advanced light curve modelling. We carried out fitting with both methods to assess robustness of results.

\subsubsection{Results and interpretation}

Figure 1 (left) shows the bolometric data of AT2017gfo compared to the best fitting Arnett model, both without (blue) and with (green) a thermalization factor (gamma-ray escape). These models have a diffusion phase lasting 1-2d, and then settle on a steady-state (short reprocessing time) tail. The most important thing here is that this tail follows closely a power law with index -1.0 to -1.3 . This is precisily the value predicted for r-process radioactivity [6]. We see this as strong confirmation of theory and definite identification of a kilonova. Note that the data beyond $8 \mathrm{~d}$ is sparse, and potential contributions beyond $2.5 \mu \mathrm{m}$ are ignored; this may explain the deviation of the data from the model. The other parameters emerging from the fit is an ejecta mass of a few hundredths of a solar mass, and a low opacity. The fit probes the $\kappa / v$ combination, and the fitting prefers the lowest allowed $\kappa\left(0.1 \mathrm{~cm}^{2} \mathrm{~g}^{-1}\right)$ and highest allowed $v(0.2 \mathrm{c})$. This is necessary to give a diffusion phase that is as short at $1-2 \mathrm{~d}$.

The right panel of Fig. 1 shows fits enforcing the opacity to $\kappa=10 \mathrm{~cm}^{2} \mathrm{~g}^{-1}$, the value predicted for lanthanide-rich ejecta $[10,19]$. Such a model cannot fit all the data well (blue line), but it may describe the later data points (green, dashed). Note though that the power law is then further away from $t^{-1.2}$, and in addition the contribution by a low-opacity component for the earlier data cannot drop away enough to avoid overproducing luminosity at later epochs.
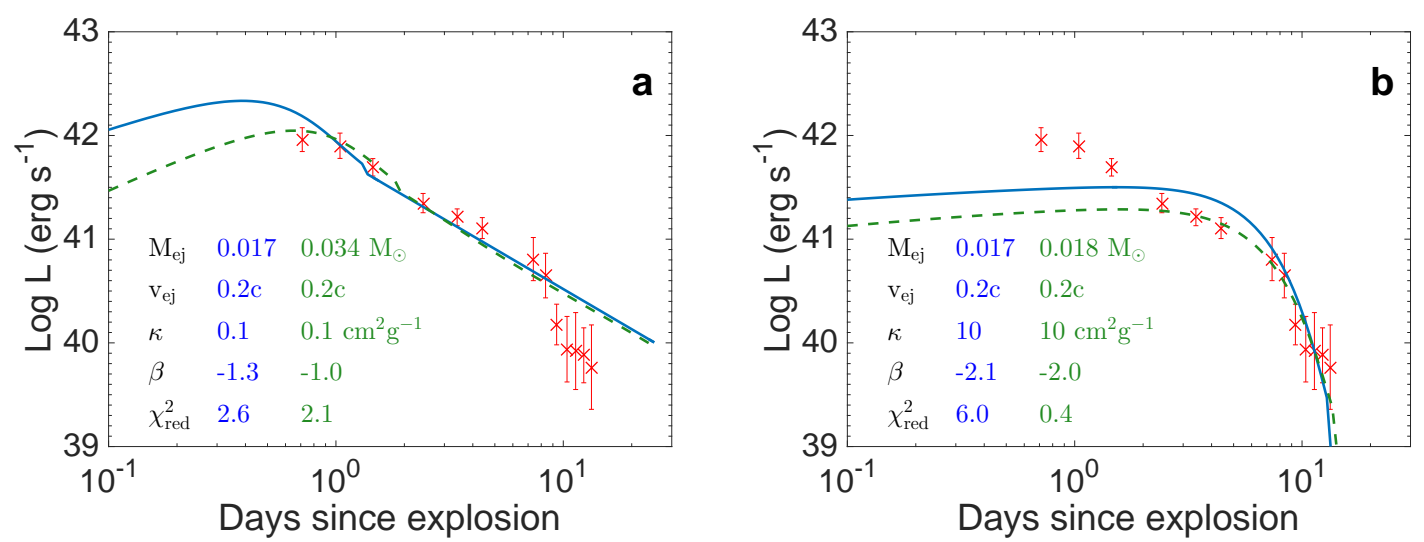

Figure 1: Bolometric light curve of AT2017gfo (red crosses) and best fitting models. Left: Opacity allowed to vary, best value is $\kappa=0.1 \mathrm{~cm}^{2} \mathrm{~g}^{-1}$. Blue solid line is model without thermalization factor, green dashed line is model with. Right: Opacity forced to $\kappa=10 \mathrm{~cm}^{2} \mathrm{~g}^{-1}$. Blue solid line is fit to all data, green dashed line is fit excluding first three points.

We also carried out a full Markov Chain Monte Carlo analysis using both Arnett and Metzger formalisms. At 1 sigma deviation levels, the model parameters are as given in table 1. 


\begin{tabular}{|c|c|c|}
\hline Parameter/Model & Arnett & Metzger \\
\hline$M\left(M_{\odot}\right)$ & $0.032-0.050$ & $0.040-0.063$ \\
$\kappa\left(\mathrm{cm}^{2} \mathrm{~g}^{-1}\right)$ & $0.16-0.63$ & $0.20-2.0$ \\
$\beta$ & $-0.9--1.5$ & $\mathrm{n} / \mathrm{a}$ \\
\hline
\end{tabular}

Table 1: Fitted range (1 sigma) for $M, \kappa$ and $\beta$ using the Arnett and Metzger formalisms.

\subsection{Spectral modelling}

The spectra were modelled using the TARDIS code [20]. Models containing Cs I and Te I were demonstrated to reproduce the main absorption/scattering features seen in the optical. While this is a tantalizing result, one should be clear that spectral formation in $\mathrm{KNe}$ is a formidable computational challenge, and all models currently suffer from insufficiency in atomic data sets being available (see discussion in [10]). If the $\mathrm{Cs}$ and Te identifications hold, they have the attractive property of belonging to the first r-process peak, which has an opacity relatively consistent with the one inferred from the light curve modelling.
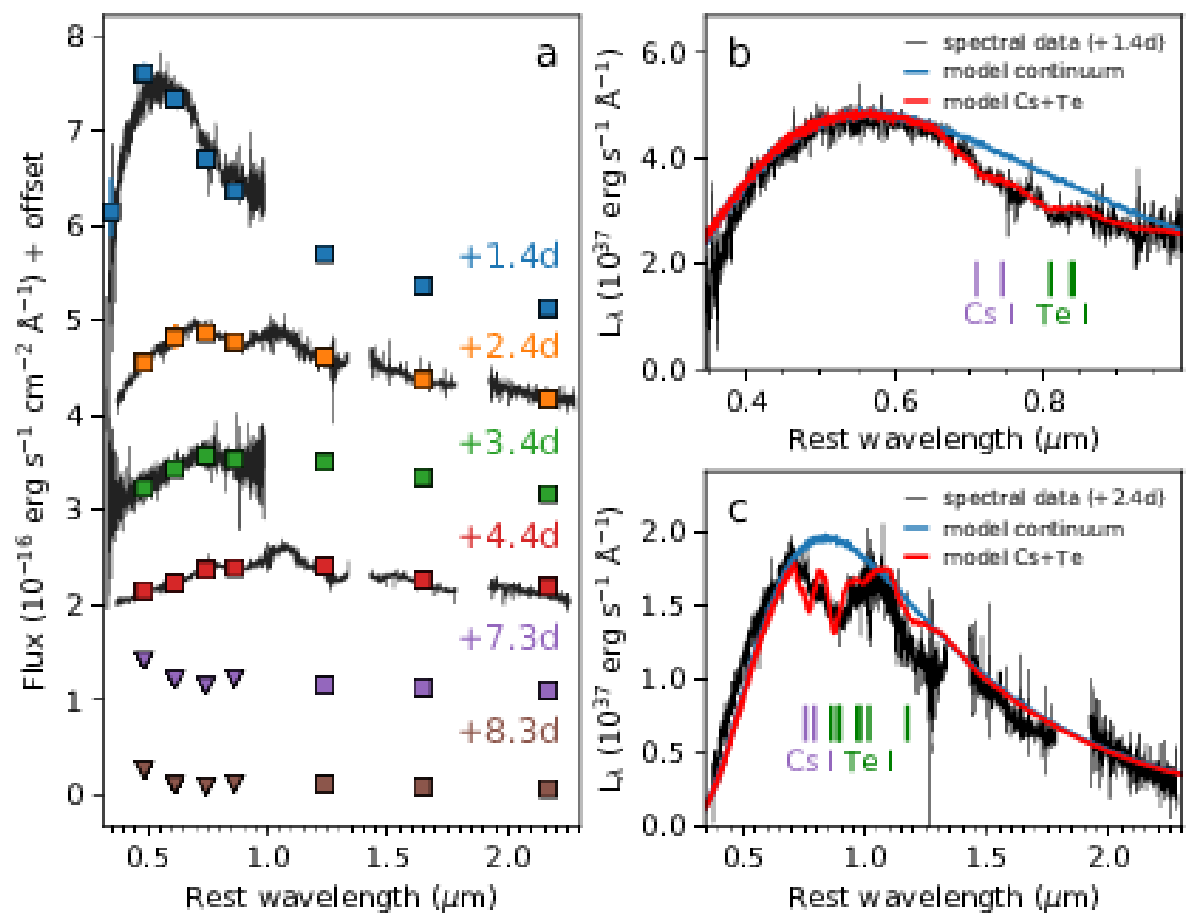

Figure 2: Spectra of AT2017gfo. The right panels shows TARDIS fits (red lines) with identification of the r-process elements $\mathrm{Cs}$ and Te.

\section{Discussion}

\subsection{A consistent picture to explain delayed GRB, low opacity, and high mass?}

The relatively high mass inferred $\left(0.04 M_{\odot}\right)$ is higher than obtained in most simulations for dynamic KN ejecta. Winds from the accretion disk, on the other hand, may become relatively 
massive. This is particularly so if the accretion disk is long-lived around a hypermassive NS that survives for $\mathrm{t} \gtrsim 1 \mathrm{~s}$ [21]. A long-lived disk produces two other effects; a delay in the formation of the short GRB (if assumed to occur once the $\mathrm{BH}$ is formed), and a higher $Y_{e}$ value of the wind material as neutrino irradiation has more time to act. This higher $Y_{e}$ in turn leads to production of lighter r-process elements which have lower opacity [21]. This scenario thus offers an attractive explanation for the 2s delay between the GWs and the GRB, the high KN mass, and its low opacity. The quantity that fits less well is the high velocity of $0.2 \mathrm{c}$; winds are typically associated with lower values $(0.05-1) \mathrm{c}$ in many simulations.

Either way, if this kind of mass is typical of $\mathrm{KNe}$, we have most likely identified the main source of r-process elements in the Universe. The galactic r-process production rate has been estimated to be around $10^{-6} M_{\odot} /$ year [22]. Then, if kilonovae are responsible for the bulk of this, their rate need to satisfy

$$
\dot{N}=2 \times 10^{-5} y r^{-1}\left(\frac{\bar{M}}{0.04 M_{\odot}}\right)^{-1}
$$

where $\bar{M}$ is the average ejecta mass. Such a rate; $1 \mathrm{KN}$ every 50,000 years per galaxy, is lower than most estimates (e.g. Kalogera et al. 2004 [23] estimates one every 3,000-50,000 yr ion the Milky Way)

\subsection{Outlook : observations}

The occurrence of a NS merger at only $40 \mathrm{Mpc}$, and the closest short GRB ever observed, so shortly after LIGO started observing currently appears like incredible luck. The merger rate has been predicted to be $0.1-1 \%$ of the core-collapse SN rate [23]. Within $40 \mathrm{Mpc}$ we have about 10 core-collapse SNe every year, so the expected time between mergers within $40 \mathrm{Mpc}$ is then 10$100 \mathrm{y}$. The situation is spookily reminiscent of SN 1998bw - the first SN detected in association with a long GRB. Also at $\sim 40 \mathrm{Mpc}$, and detected soon after the first space-born gamma ray detectors CGRO and Beppo-SAX were in place, no other similarly nearby event has been detected since, although about a handful at much larger distances have been discovered.

The similarities with SN 1998bw do not stop here. GRB980425 was an unusually weak (long) GRB, and the dim afterglow allowed the underlying SN to be clearly seen. The fundamental reason that AT2017gfo could be clearly detected was also that the GRB afterglow was unusually weak. The situation finds a quite natural explanation in that nearby off-axis events can give weaker, but still detectable, gamma emission compared to larger distance events [24], which we more easily see if they are on axis and reach maximum beaming. But the beaming does not affect the nonrelativistic SN/KN transient much. Thus, it becomes much more difficult to see this transient at higher distance; it gets swamped by the afterglow. This is probably precisely what happened in the cases of GRB 080503 [25] and GRB 130603B [26, 27]. While there are bumps in the GRB afterglow light curves possibly indicating presence of $\mathrm{KNe}$, the afterglows are strong and the $\mathrm{KN}$ signals cannot be clearly extracted.

This all suggests that interpretation of $\mathrm{KNe}$ is intrinsically linked to understanding the GRB afterglow. It may also mean that AT2017gfo could remain a unique event for a long time. But it may also be that the theoretical rates are too low, and that we will see a relatively high frequency of similar events in the nearby $(\lesssim 100 \mathrm{Mpc}$ ) Universe. The fraction of massive stars in relatively 
close binaries have recently been dramatically revised upwards [28], which could indicate such a scenario.

\subsection{Outlook: Modelling}

Regarding modelling, simulating the light curve and spectral formation in KN ejecta is a complex process still in its infancy, really begun only in 2010 [6]. It will take several years before atomic data calculations progress to the point that reliable light curves can be calculated, and even longer before accurate spectra can be calculated. The spectral simulations also need to consider complications in the form of 3D effects, breakdown of the Sobolev approximation, NLTE, and perhaps even processes such as r-process dust formation. However exciting first steps have been taken $[6,10,19]$. We are clearly at the beginning of a new and exciting era in transient astronomy. Despite all the caveats and uncertain aspects of the models, both in hydrosimulations and radiative transfer, AT2017gfo is to some extent a fantastic success for theory, appearing largely as predicted and confirming the basics theories of neutron star mergers having been worked out over the last 30 years.

\section{References}

[1] B. Paczynski, Gamma-ray bursters at cosmological distances, ApJ 308 (Sept., 1986) L43-L46.

[2] J. M. Lattimer and D. N. Schramm, Black-hole-neutron-star collisions, ApJ 192 (Sept., 1974) L145-L147.

[3] D. Eichler, M. Livio, T. Piran and D. N. Schramm, Nucleosynthesis, neutrino bursts and gamma-rays from coalescing neutron stars, Nature 340 (July, 1989) 126-128.

[4] S. Rosswog, U. Feindt, O. Korobkin, M.-R. Wu, J. Sollerman, A. Goobar et al., Detectability of compact binary merger macronovae, Classical and Quantum Gravity 34 (May, 2017) 104001, [1611.09822].

[5] L.-X. Li and B. Paczyński, Transient Events from Neutron Star Mergers, ApJ 507 (Nov., 1998) L59-L62, [astro-ph/9807272].

[6] B. D. Metzger, G. Martínez-Pinedo, S. Darbha, E. Quataert, A. Arcones, D. Kasen et al., Electromagnetic counterparts of compact object mergers powered by the radioactive decay of r-process nuclei, MNRAS 406 (Aug., 2010) 2650-2662, [1001. 5029].

[7] B. P. Abbott, R. Abbott, T. D. Abbott, F. Acernese, K. Ackley, C. Adams et al., GW170817: Observation of Gravitational Waves from a Binary Neutron Star Inspiral, Physical Review Letters 119 (Oct., 2017) 161101, [1710.05832].

[8] S. J. Smartt, T.-W. Chen, A. Jerkstrand, M. Coughlin, E. Kankare, S. A. Sim et al., A kilonova as the electromagnetic counterpart to a gravitational-wave source, ArXiv e-prints (Oct., 2017), [1710.05841].

[9] S. A. Colgate and R. H. White, The Hydrodynamic Behavior of Supernovae Explosions, ApJ 143 (Mar., 1966) 626.

[10] D. Kasen, N. R. Badnell and J. Barnes, Opacities and Spectra of the r-process Ejecta from Neutron Star Mergers, ApJ 774 (Sept., 2013) 25, [1303. 5788]. 
[11] M.-R. Wu, R. Fernández, G. Martínez-Pinedo and B. D. Metzger, Production of the entire range of r-process nuclides by black hole accretion disc outflows from neutron star mergers, MNRAS 463 (Dec., 2016) 2323-2334, [1607 .05290].

[12] J. Greiner, W. Bornemann, C. Clemens, M. Deuter, G. Hasinger, M. Honsberg et al., GROND - $a$ 7-Channel Imager, PASP 120 (Apr., 2008) 405, [0801. 4801].

[13] P. A. Evans, S. B. Cenko, J. A. Kennea, S. W. K. Emery, N. P. M. Kuin, O. Korobkin et al., Swift and NuSTAR observations of GW170817: detection of a blue kilonova, ArXiv e-prints (Oct., 2017), [1710.05437].

[14] W. D. Arnett, Analytic solutions for light curves of supernovae of Type II, ApJ 237 (Apr., 1980) 541-549.

[15] W. D. Arnett, Type I supernovae. I - Analytic solutions for the early part of the light curve, ApJ 253 (Feb., 1982) 785-797.

[16] E. Chatzopoulos, J. C. Wheeler and J. Vinko, Generalized Semi-analytical Models of Supernova Light Curves, ApJ 746 (Feb., 2012) 121, [1111.5237].

[17] C. Inserra, S. J. Smartt, A. Jerkstrand, S. Valenti, M. Fraser, D. Wright et al., Super-luminous Type Ic Supernovae: Catching a Magnetar by the Tail, ApJ 770 (June, 2013) 128, [1304 . 3320].

[18] B. D. Metzger, Kilonovae, Living Reviews in Relativity 20 (May, 2017) 3, [1 610.09381$].$

[19] M. Tanaka and K. Hotokezaka, Radiative Transfer Simulations of Neutron Star Merger Ejecta, ApJ 775 (Oct., 2013) 113, [1306.3742].

[20] W. E. Kerzendorf and S. A. Sim, A spectral synthesis code for rapid modelling of supernovae, MNRAS 440 (May, 2014) 387-404, [1 401.5469 ].

[21] B. D. Metzger and R. Fernández, Red or blue? A potential kilonova imprint of the delay until black hole formation following a neutron star merger, MNRAS 441 (July, 2014) 3444-3453, [14 02 . 4803 ].

[22] Y.-Z. Qian, Supernovae versus Neutron Star Mergers as the Major R-Process Sources, ApJ 534 (May, 2000) L67-L70, [astro-ph/ 0003242 ].

[23] V. Kalogera, C. Kim, D. R. Lorimer, M. Burgay, N. D’Amico, A. Possenti et al., The Cosmic Coalescence Rates for Double Neutron Star Binaries, ApJ 601 (Feb., 2004) L179-L182.

[24] J. M. Burgess, J. Greiner, D. Begue, D. Giannios, F. Berlato and V. M. Lipunov, Viewing short Gamma-ray Bursts from a different angle, ArXiv e-prints (Oct., 2017), [1710 . 05823].

[25] D. A. Perley, B. D. Metzger, J. Granot, N. R. Butler, T. Sakamoto, E. Ramirez-Ruiz et al., GRB 080503: Implications of a Naked Short Gamma-Ray Burst Dominated by Extended Emission, ApJ 696 (May, 2009) 1871-1885, [0811. 1044 ].

[26] E. Berger, W. Fong and R. Chornock, An r-process Kilonova Associated with the Short-hard GRB 130603B, ApJ 774 (Sept., 2013) L23, [1306.3960].

[27] N. R. Tanvir, A. J. Levan, A. S. Fruchter, J. Hjorth, R. A. Hounsell, K. Wiersema et al., A 'kilonova' associated with the short-duration $\gamma$-ray burst GRB 130603B, Nature $\mathbf{5 0 0}$ (Aug., 2013) 547-549, [1306.4971].

[28] H. Sana, S. E. de Mink, A. de Koter, N. Langer, C. J. Evans, M. Gieles et al., Binary Interaction Dominates the Evolution of Massive Stars, Science 337 (July, 2012) 444, [12 07 . 6397]. 